\title{
Gene expression profiling analysis of keloids with and without hydrocortisone treatment
}

\author{
HONGYI WANG, LIANGLIANG QUAN, JIULONG LIANG, JIE SHI, TAO QIU, \\ YE ZHANG, YANG WANG, QIANG HUI, YU ZHANG and KAI TAO \\ Department of Plastic Surgery, General Hospital of Shenyang Military Area \\ Command, PLA, Shenyang, Liaoning 110016, P.R. China
}

Received February 25, 2016; Accepted February 24, 2017

DOI: $10.3892 /$ etm. 2017.5263

\begin{abstract}
The present study aimed to investigate the genetic effects of hydrocortisone (HC) treatment on keloids and screen medicines to be used in a combination therapy of keloids with HC. The dataset GSE7890 was downloaded from Gene Expression Omnibus. It contained data regarding 4 fibroblast samples from normal scar tissue and 5 samples from keloid tissue with $\mathrm{HC}$ treatment, as well as 5 samples from normal scar and 5 samples from keloids without $\mathrm{HC}$ treatment. Following the identification of differentially expressed genes (DEGs), the functions of these DEGs were analyzed by Gene Ontology (GO) and pathway enrichment analyses. Furthermore, adverse effects of HC were identified using WebGestalt. Additionally, candidate small molecule drugs associated with keloids were selected from a connectivity map database. A total of 166 and 41 DEGs, with and without HC treatment respectively, were only present in dermal fibroblasts from keloids (termed genesets A and B, respectively). A set of 26 DEGs was present following both treatments (geneset $\mathrm{C}$ ). A number of DEGs in geneset $\mathrm{B}$ (COL18Al and JAG1) were associated with endothelial cell differentiation. However, in genesets $\mathrm{A}$ and $\mathrm{C}$, certain genes (CCNB1 and $C C N B 2)$ were involved in the cell cycle and p53 signaling pathways, and a number of genes (ILIRI and $C O L 1 A 1)$ were associated with bone loss. Additionally, numerous small molecule drugs (including acemetacin) were associated with keloids. Thus, it has been determined that HC may treat keloids by targeting genes associated to endothelial cell differentiation (COL18Al and JAGl). However, $\mathrm{HC}$ has a number of adverse effects, including bone loss. Acemetacin may be applied in a combination therapy, along with $\mathrm{HC}$, to treat keloids.
\end{abstract}

Correspondence to: Dr Kai Tao, Department of Plastic Surgery, General Hospital of Shenyang Military Area Command, PLA, 83 Wenhua Road, Shenhe, Shenyang, Liaoning 110016, P.R. China E-mail:ktaoai@163.com

Key words: keloid, hydrocortisone, differentially expressed gene, adverse effect, small molecule drug

\section{Introduction}

Keloids are benign fibrotic tumors of the dermis that form during a prolonged wound healing process (1). Keloids exhibit aggressive dermal growth beyond the boundaries of the original margins of wounds, causing pain, pruritis and contractures. They are a cosmetic and psychological burden to patients (2).

The effect of one type of glucocorticoid, hydrocortisone (HC), on keloids has previously been investigated. HC is known to alter the chemistry and morphology of connective tissue cells and to hinder the production of intercellular substances, including collagenous fiber and glycosaminoglycan (3). It may also decrease the maximum density of keloid-derived fibroblasts (4), increase proline transport (5), lower prolyl hydroxylase activity, reduce the rate of collagen synthesis (6), enhance apoptosis rates (7) and diminish hyaluronan accumulation (8).

Microarray studies identify a broad spectrum of differentially regulated genes in biological samples under genomic sequencing, cloning, cDNA or PCR approaches (9). In 2008, Smith et al (10) conducted a bioinformatic analysis on RNA obtained from fibroblasts cultured from normal scars and keloids grown in the absence and presence of $\mathrm{HC}$. The results indicated that there was elevated expression of a number of insulin-like growth factor (IGF)-binding and IGF-binding related proteins, in addition to decreased expression of a set of Wnt pathway inhibitors and numerous interleukin (IL)-1-inducible genes. Furthermore, it was observed that IGF binding protein (IGFBP)-3 and connective tissue growth factor (CTGF) were associated with the increase of fibroblast and collagen deposition and were overexpressed in keloid fibroblasts only in the presence of $\mathrm{HC}$, suggesting that glucocorticoid resistance of $\mathrm{HC}$ is involved in the pathogenesis of keloids formation (10). However, the adverse effects of $\mathrm{HC}$ during its treatment on keloids and the drugs that may potentially be used to weaken or reverse these adverse effects, remain unknown.

The present study used the microarray data collected by Smith et al (10) to identify differentially expressed genes (DEGs) in fibroblasts cultured from keloids treated with or without HC. A Gene Ontology (GO) enrichment analysis was performed on DEGs, which were potentially associated with 
the positive efficacy of $\mathrm{HC}$, and a pathway enrichment analysis for DEGs that may be associated with adverse effects of HC was completed. Furthermore, the adverse effects of HC were analyzed and small molecule drugs that may reduce the occurrence of adverse effects were screened from the connectivity map (CMAP) database. The aim of the present study was to elucidate the molecular mechanisms of $\mathrm{HC}$ treatment on keloids and provide novel information for the clinical treatment of this disease.

\section{Materials and methods}

Affymetrix microarray data. The gene expression profile data of GSE7890 (10) was downloaded from the Gene Expression Omnibus (GEO; http://www.ncbi.nlm.nih.gov/geo/) database, which was based on the platform of GPL570 [HG-U133 Plus_2] Affymetrix Human Genome U133 Plus 2.0 Array (Affymetrix Inc., Santa Clara, CA, USA). A total of 19 primary cultures of dermal fibroblast samples were included in this dataset, including 4 samples from normal scar tissue and 5 samples from keloids treated with $1.5 \mu \mathrm{M} \mathrm{HC}$; as well as 5 samples from normal scar and 5 samples from keloids that did not undergo $\mathrm{HC}$ treatment.

Affymetrix CEL files and the probe annotation files were downloaded and the gene expression data of all samples were preprocessed via background correction, quantile normalization and probe summarization using the Affy software (version 1.30.0) packageofBioconductor(availableathttp://www.bioconductor.org/packages/2.8/bioc/html/affy.html) (11).

DEGs screening. Linear Models for Microarray Data package (version 3.22.7) (12) of Bioconductor (available at http://www .bioconductor.org/packages/3.0/bioc/html/limma.html) was used to identify genes that were differentially expressed in dermal fibroblasts from keloids and those from normal scars. The raw P-value was adjusted into False Discovery Rate (FDR) by the Bonferroni method (13) in a multtest package (version 2.20.0) of Bioconductor (http://www.bioconductor.org/packages $/ 2.14 / \mathrm{bioc} / \mathrm{html} / \mathrm{multtest}$.html). The $\mid \log _{2} \mathrm{FC}$ (fold change) $\mid>1$ and FDR $<0.05$ were selected as the cut-off criteria.

Functional classification of DEGs. GO functional enrichment analysis of DEGs was performed to interpret their biological significance, via the Database for Annotation, Visualization and Integrated Discovery (http://david.abcc.ncifcrf.gov/) (14). $\mathrm{P}<0.01$ was used as the cut-off criterion.

Pathway enrichment analysis of DEGs was performed using a Kegg Orthology Based Annotation System, version 2.0 (http://kobas.cbi.pku.edu.cn) (15). $\mathrm{P}<0.05$ was used as the cut-off criterion.

Identification of potential diseases associated with DEGs. Potential diseases associated with the DEG genesets A and $\mathrm{C}$ were identified using the WEB-based GEne SeT AnaLysis Toolkit (WebGestalt; www.webgestalt.org) (16) with a cut-off criterion of $\mathrm{P}<0.05$.

Prediction of small molecule drugs. CMAP database (www.connectivitymap.org/cmap) (17) was used to predict candidate small molecule drugs targeting DEGs genesets A and C. An enrichment score between 0.9-1 was selected as the cut-off criterion. The closer to -1 the enrichment score was, the stronger the effect of the drug on the disease.

\section{Results}

Identification of DEGs. A total of 192 DEGs with HC treatment (50 upregulated and 142 downregulated) and 67 DEGs without $\mathrm{HC}$ treatment (15 upregulated and 52 downregulated) were screened from dermal fibroblasts in keloids and compared with normal scar tissue.

Among them, 166 DEGs were only present in the dermal fibroblasts from keloids treated with HC (geneset A), 41 DEGs were only present in dermal fibroblasts from keloids with no HC treatment (geneset B) and 26 DEGs were common in dermal fibroblasts from keloids with and without $\mathrm{HC}$ treatment (geneset C; Fig. 1).

Functional annotation of DEG. To investigate the potential molecular functions in keloid fibroblasts affected by $\mathrm{HC}$, a GO functional enrichment analysis of the DEGs in geneset B, which were potentially associated with the positive efficacy of HC, was performed. According to the GO functional enrichment analysis, DEGs in geneset B were significantly enriched in $9 \mathrm{GO}$ terms (all $\mathrm{P}<0.01$ ), including endothelial cell differentiation (COL18A1, HOXB5 and JAGI) and regulation of cell proliferation (DLCl, COL18A1 and JAGl; Table I). Furthermore, $21.28 \%$ of DEGs were associated with the regulation of cell proliferation and $8.51 \%$ of DEGs with endothelial cell differentiation (Fig. 2).

To identify the potential dysregulation pathways associated with $\mathrm{HC}$ treatment and those that were unaffected by $\mathrm{HC}$, a pathway enrichment analysis of the DEGs in geneset A (specifically, novel DEGs following HC treatment) and genes in geneset $\mathrm{C}$ (namely, genes that were not affected by $\mathrm{HC}$ ), was performed. Based on this pathway enrichment analysis, the DEGs in genesets $\mathrm{A}$ and $\mathrm{C}$ were significantly enriched in two pathways: Cell cycle (CCNB1, MAD2L1 and BUB1) and p53 signaling pathway (CCNB1, RRM2 and PERP; Table II; $\mathrm{P}<0.05)$.

Potential diseases associated with DEGs in genesets A and C. To investigate the adverse effects of $\mathrm{HC}$ during its treatment on keloids, potential diseases associated with DEGs in genesets A and $\mathrm{C}$ were identified. It was determined that the newly added DEGs in dermal fibroblasts from keloids following $\mathrm{HC}$ treatment and genes that were not altered by $\mathrm{HC}$ were associated with two diseases: Bone loss (ILIRI, HSPA2 and COL1A1) and osteoarthritis (ILIRI, IGFBP7 and COL1A1; Table III).

Small molecule drugs targeting DEGs in genesets $A$ and $C$. To further investigate potential drugs that are able to weaken or eliminate the adverse effects of $\mathrm{HC}$, small molecule drugs targeting DEGs in genesets $\mathrm{A}$ and $\mathrm{C}$ were identified. A total of 9 small molecule drugs were selected from the CMAP database, including acemetacin, scriptaid and alsterpaullone. Among them, acemetacin has a negative score and the lowest $\mathrm{P}$-value $(\mathrm{P}=0.00002)$, indicating it had the strongest effect on the adverse effects of $\mathrm{HC}$ on gene expression (Table IV). 
Table I. Enriched GO terms for differentially expressed genes in geneset B.

\begin{tabular}{|c|c|c|c|c|}
\hline Term & Description & Count & P-value & Genes \\
\hline GO:0045446 & Endothelial cell differentiation & 4 & $\mathrm{P}<0.001^{\mathrm{a}}$ & COL18A1, HOXB5, JAG1, NR2F2 \\
\hline GO:0042127 & Regulation of cell proliferation & 10 & $\mathrm{P}<0.001^{\mathrm{a}}$ & $\begin{array}{l}\text { DLC1, COL18A1, NCK2, TBX3, NKX3-1, } \\
\text { PTN, JAG1, PPAP } 2 A, C L E C 11 A, I G F B P 5\end{array}$ \\
\hline GO:0008285 & Negative regulation of cell proliferation & 6 & $0.001937^{\mathrm{a}}$ & $\begin{array}{l}D L C 1, C O L 18 A 1, N C K 2, N K X 3-1, P P A P 2 A, \\
I G F B P 5\end{array}$ \\
\hline GO:0060429 & Epithelium development & 5 & $0.002412^{\mathrm{a}}$ & DLC1, COL18A1, HOXB5, JAG1, NR2F2 \\
\hline GO:0001568 & Blood vessel development & 5 & $0.003177^{\mathrm{a}}$ & COL18A1, TBX3, MMP19, JAG1,NR2F2 \\
\hline GO:0001944 & Vasculature development & 5 & $0.003465^{\mathrm{a}}$ & COL18A1, TBX3, MMP19, JAG1, NR2F2 \\
\hline GO:0030855 & Epithelial cell differentiation & 4 & $0.004833^{\mathrm{a}}$ & COL18A1, HOXB5, JAG1, NR2F2 \\
\hline GO:0009952 & Anterior/posterior pattern formation & 4 & $0.005134^{\mathrm{a}}$ & $T B X 3, H O X B 5, N K X 3-1, N R 2 F 2$ \\
\hline GO:0030334 & Regulation of cell migration & 4 & $0.008627^{\mathrm{a}}$ & DLC1, COL18A1, JAG1, IGFBP5 \\
\hline
\end{tabular}

${ }^{\mathrm{a}} \mathrm{P}<0.01$. Geneset B represents the differentially expressed genes specifically present in dermal fibroblasts from keloids without hydrocortisone treatment, specifically, genes that were not differentially expressed following HC treatment. GO, Gene Ontology.

Table II. Results of pathway enrichment analysis for differentially expressed genes in genesets A and C.

\begin{tabular}{llcll}
\hline Term & \multicolumn{1}{c}{ Description } & Count & P-value & \multicolumn{1}{c}{ Genes } \\
\hline hsa04110 & Cell cycle & 9 & $4.29 \mathrm{E}-05^{\mathrm{a}}$ & $\begin{array}{l}\text { CCNB1, MAD2L1, CCNB2, DBF4, BUB1, TTK, CDC20, } \\
\text { CDC25C, CCNA2 } \\
\text { hsa04115 }\end{array}$ \\
p53 signaling pathway & 5 & $0.005598^{\mathrm{a}}$ & CCNB1, CCNB2, RRM2, PERP, PTEN
\end{tabular}

${ }^{a} \mathrm{P}<0.01$. Geneset A represents the differentially expressed genes present in dermal fibroblasts from keloids with hydrocortisone treatment, specifically, novel differentially expressed genes following hydrocortisone treatment. Geneset $\mathrm{C}$ represents the differentially expressed genes that were not affected by hydrocortisone treatment.

\section{Discussion}

Keloids are benign fibrotic tumors of the dermis and are a cosmetic and psychological burden to patients (2). In the present study, 192 and 67 DEGs were screened from dermal fibroblasts in keloids with and without $\mathrm{HC}$ treatment, respectively, and compared with gene expression in normal scar tissue. According to the GO functional enrichment analysis for DEGs only present in dermal fibroblasts from keloids without $\mathrm{HC}$ treatment, namely those expressed normally following $\mathrm{HC}$ treatment, a set of genes (COL18A1 and JAG1) were significantly associated with endothelial cell differentiation and regulation of cell proliferation $(\mathrm{P}<0.001)$.

COL18A1 encodes the alpha chain of type XVIII collagen, which is an inhibitor of angiogenesis (18). It has demonstrated that collagen XVIII levels are downregulated in keloid patients compared with normal controls (19). Inhibition of collagen synthesis was observed following the addition of higher concentrations of HC (20). JAG1 is one of Notch ligands from the Notch family (21). Increased expression of JAG1 and Notch receptors has been observed in keloid fibroblasts and the inhibition of Notch signaling via JAGl knockdown led to the inhibition of proliferation, migration and invasion properties of keloid fibroblasts (22). Furthermore, the endothelial cells within blood vessels in keloids are somewhat rounded and projected into the lumen of the vessel (23); this has been

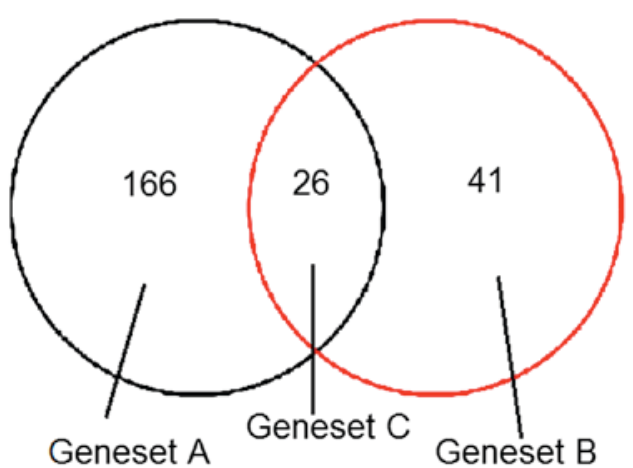

Figure 1. Venn diagram for the differentially expressed genes in dermal fibroblasts from keloids with and without hydrocortisone treatment, compared with normal scar tissue. Geneset A represents genes only present in dermal fibroblasts of keloids that have undergone $\mathrm{HC}$ treatment; geneset $\mathrm{B}$ represents genes only present in dermal fibroblasts of keloids without HC treatment; and geneset $\mathrm{C}$ represents genes present in dermal fibroblasts both with and without $\mathrm{HC}$ treatment. $\mathrm{HC}$, hydrocortisone.

speculated as critical to facilitate the development and maintenance of keloids (24). Vascular endothelial growth factor, which promotes angiogenesis and enhances endothelial cell survival (25), is abundantly produced in keloids $(26,27)$. As DEGs in geneset B were not present in the samples following $\mathrm{HC}$ treatment, these genes were considered to be potentially 


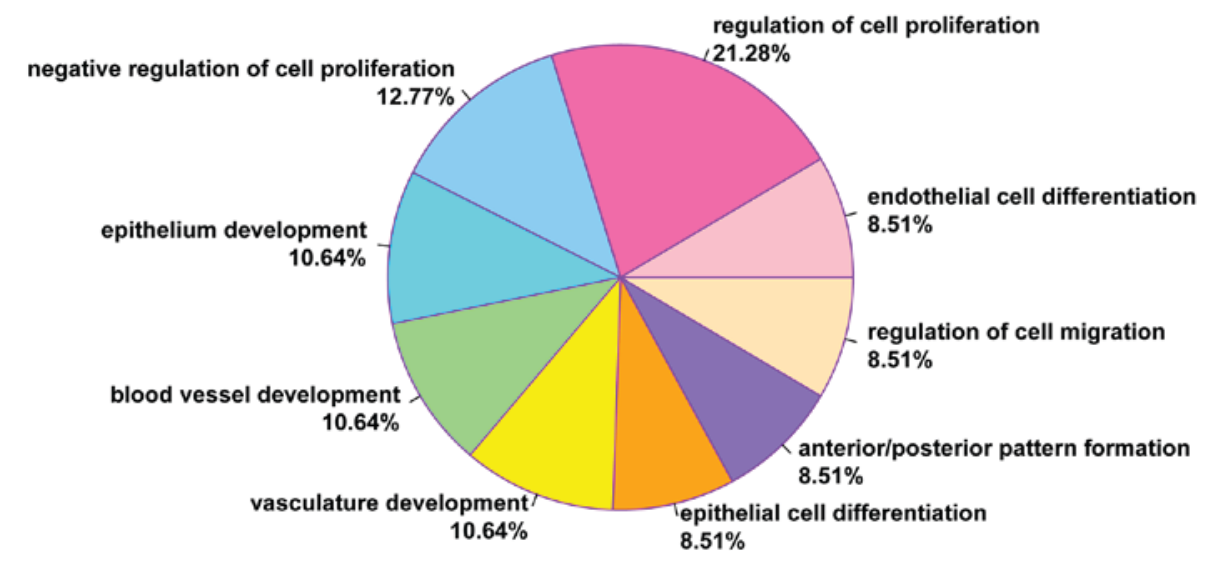

Figure 2. Pie Chart for Gene Ontology functional classification of genes only differentially expressed in dermal fibroblasts from keloids without HC treatment, compared with normal scar tissue. Genes presented in this fig. are differentially expressed in keloids without HC treatment, but expressed normally following HC treatment. HC, hydrocortisone.

Table III. Potential diseases associated with differentially expressed genes in genesets $\mathrm{A}$ and $\mathrm{C}$.

\begin{tabular}{lcc}
\hline Disease & P-value & Genes \\
\hline Bone loss & $0.002593^{\mathrm{a}}$ & IL1R1, HSPA2, COL1A1 \\
Osteoarthritis & $0.033811^{\mathrm{a}}$ & IL1R1,IGFBP7,COL1A1,CALM1
\end{tabular}

${ }^{\mathrm{a}} \mathrm{P}<0.05$. Geneset $\mathrm{A}$ represents the differentially expressed genes present in dermal fibroblasts from keloids with hydrocortisone treatment, specifically, novel differentially expressed genes following hydrocortisone treatment. Geneset $\mathrm{C}$ represents the differentially expressed genes that were not affected by hydrocortisone treatment.

associated with the positive efficacy of HC. Therefore, HC may serve a key role in the treatment of keloids by targeting genes associated with endothelial cell differentiation and regulation of cell proliferation (COL18Al and JAG1).

The pathway enrichment analysis for the DEGs in genesets $\mathrm{A}$ and $\mathrm{C}$ revealed that certain DEGs $(C C N B 1$ and $C C N B 2)$ were significantly enriched in the cell cycle and p53 signaling pathways $(\mathrm{P}<0.05)$. Keloids are characterized by aggressive dermal growth beyond the boundaries of the original margins of a wound (2), which is dependent on the ectopic cell cycle. A previous study demonstrated that mRNAs associated with cell cycle suppression, such as cyclin B1, were detected in the bottom region of keloids (28). Furthermore, CCNB1 gene expression is reduced by low-dose 5-fluorouracil in treated keloid fibroblasts (29). The p53 tumor suppressor acts as a transcription factor and has a central function in controlling apoptosis (30). p53 levels are higher in keloids compared with normal scar tissues (31). Therefore, the current study demonstrated that $C C N B 1$ and $C C N B 2$ are involved in the cell cycle and p53 signaling pathways may be essential in the development of keloids. However, the current results indicated that the expression of these genes was altered following $\mathrm{HC}$ treatment, and did not change in the samples without $\mathrm{HC}$ treatment. Thus, it is speculated that the alterations of $C C N B 1$ and $C C N B 2$ may contribute to keloids via dysregulation of cell cycle and p53 signaling pathways.
Table IV. Small molecule drugs potentially used to treat the diseases associated with differentially expressed genes in genesets $\mathrm{A}$ and $\mathrm{C}$.

\begin{tabular}{lcc}
\hline Small molecule drug & Enrichment & P-value \\
\hline Acemetacin & -0.925 & $0.00002^{\mathrm{a}}$ \\
Scriptaid & 0.912 & $0.00142^{\mathrm{a}}$ \\
Alsterpaullone & 0.923 & $0.00094^{\mathrm{a}}$ \\
Mycophenolic acid & 0.957 & $0.0001^{\mathrm{a}}$ \\
MG-262 & 0.962 & $0.00008^{\mathrm{a}}$ \\
Trifluridine & 0.975 & $<0.001$ \\
Camptothecin & 0.975 & $0.00004^{\mathrm{a}}$ \\
Ciclopirox & 0.987 & $<0.001$ \\
MS-275 & 0.998 & $0.00002^{\mathrm{a}}$ \\
\hline
\end{tabular}

${ }^{\mathrm{a}} \mathrm{P}<0.05$. Geneset Arepresents the differentially expressed genes present in dermal fibroblasts from keloid with hydrocortisone treatment, specifically, novel differentially expressed genes following hydrocortisone treatment. Geneset $\mathrm{C}$ represents the differentially expressed genes that are not affected by hydrocortisone. The closer to -1 the enrichment score is, the stronger the effect of the drug on the diseases.

A number of diseases, such as bone loss, were predicted to be associated with DEGs in keloid fibroblasts treated with HC (ILIRI and COLIAI). Bone loss is one of the most devastating adverse effects of glucocorticoids as it results in the inhibition of calcium transport and the impairment of osteoblast function (32). A previous study has reported that long-term treatment of secondary hypocortisolism with a high replacement dose of hydrocortisone $(30 \mathrm{mg} /$ day $)$ induces bone loss (33). ILIRI encodes the type I interleukin 1 receptor. It has been demonstrated that IL-1 production may accelerate bone loss in postmenopausal women (34). The interleukin-1 receptor antagonist (IL-1ra) may decrease bone loss and bone resorption in ovariectomized rats (35) and it has been reported that a gene polymorphism of IL-1ra is associated with bone mineral density and osteoporosis in postmenopausal women (36). Additionally, it has been 
demonstrated that COLIAI (encoding collagen, type I, alpha 1) Sp1 alleles are associated with a modest reduction in bone mineral density and a markedly increased risk of osteoporotic fracture, particularly vertebral fracture (37). Previously, it was demonstrated that the crosslinking process of collagen is important in bone strength, osteogenesis imperfecta and osteoporosis (38). Therefore, the present study hypothesized that $\mathrm{HC}$ may contribute to bone loss during treatment with keloids, through certain genes including ILIRI and COLIAI.

Although $\mathrm{HC}$ has been successfully used to treat keloids, it also induces certain adverse effects, such as arthralgia (39). In the present study, 9 small molecule drugs were identified by the CMAP database and acemetacin was predicted to be the most effective drug at reducing the adverse effects of HC. It has previously been demonstrated that acemetacin may treat arthralgia effectively (40). Therefore, combining acemetacin with $\mathrm{HC}$ may be a novel therapeutic method of treating keloids, thus reducing the adverse effects of $\mathrm{HC}$ treatment.

Despite the aforementioned results, there were a number of limitations in the present study. Confirmation by experiment of these predictions is necessary. Future in vivo studies are required to detect the expression of genes associated with endothelial cell differentiation and regulation of cell proliferation. Furthermore, the efficacy of acemetacin-HC combination treatment compared with $\mathrm{HC}$ treatment alone requires evaluation.

In conclusion, the present study suggests that $\mathrm{HC}$ treats keloids effectively by targeting COL18A1 and JAGl, which are associated with endothelial cell differentiation. However, the cell cycle and p53 signaling pathways in keloids do not return to normal following treatment with $\mathrm{HC}$. A number of adverse effects such as bone loss, which involves ILIRI and COLIAI, may occur during the therapeutic process of $\mathrm{HC}$ treatment. Additionally, it was predicted that acemetacin may be used in combination with HC to treat keloids, although further studies are required to demonstrate its efficacy. These findings may contribute to the understanding of mechanisms involved in $\mathrm{HC}$ treatment on keloids and provide candidates for subsequent validation and further study.

\section{References}

1. Marneros AG, Norris JE, Watanabe S, Reichenberger E and Olsen BR: Genome scans provide evidence for keloid susceptibility loci on chromosomes $2 \mathrm{q} 23$ and $7 \mathrm{p} 11$. J Invest Dermatol 122: 1126-1132, 2004.

2. Fong CY, Biswas A, Subramanian A, Srinivasan A, Choolani M and Bongso A: Human keloid cell characterization and inhibition of growth with human Wharton's jelly stem cell extracts. J Cell Biochem 115: 826-838, 2014.

3. Asboe-Hansen G, Brodthagen $\mathrm{H}$ and Zachariae L: Treatment of keloids with topical injections of hydrocortisone acetate. AMA Arch Derm 73: 162-165, 1956

4. Russell JD, Russell SB and Trupin KM: Differential effects of hydrocortisone on both growth and collagen metabolism of human fibroblasts from normal and keloid tissue. J Cell Physiol 97: 221-229, 1978

5. Russell SB, Russell JD and Trupin JS: Alteration of amino acid transport by hydrocortisone. Different effects in human fibroblasts derived from normal skin and keloid. J Biol Chem 257: 9525-9531, 1982.

6. Trupin JS, Russell SB and Russell JD: Variation in prolyl hydroxylase activity of keloid-derived and normal human fibroblasts in response to hydrocortisone and ascorbic acid Coll Relat Res 3: 13-23, 1983.
7. Ladin DA, Hou Z, Patel D, McPhail M, Olson JC, Saed GM and Fivenson DP: p53 and apoptosis alterations in keloids and keloid fibroblasts. Wound Repair Regen 6: 28-37, 1998.

8. Meyer LJ, Russell SB, Russell JD, Trupin JS, Egbert BM, Shuster S and Stern R: Reduced hyaluronan in keloid tissue and cultured keloid fibroblasts. J Investig Dermatol 114: 953-959, 2000.

9. Mantripragada KK, Buckley PG, Diaz de Ståhl T and Dumanski JP: Genomic microarrays in the spotlight. Trends Genet 20: 87-94, 2004.

10. Smith JC, Boone BE, Opalenik SR, Williams SM and Russell SB: Gene profiling of keloid fibroblasts shows altered expression in multiple fibrosis-associated pathways. J Invest Dermatol 128: 1298-1310, 2008

11. Seo J and Hoffman EP: Probe set algorithms: Is there a rational best bet? BMC Bioinformatics 7: 395, 2006.

12. Smyth GK: Linear models and empirical bayes methods for assessing differential expression in microarray experiments. Stat Appl Genet Mol Biol 3: Article3, 2004.

13. Bland JM and Altman DG: Multiple significance tests: The Bonferroni method. BMJ 310: 170, 1995.

14. Huang da W, Sherman BT and Lempicki RA: Systematic and integrative analysis of large gene lists using DAVID bioinformatics resources. Nat Protoc 4: 44-57, 2009.

15. Xie C, Mao X, Huang J, Ding Y, Wu J, Dong S, Kong L, Gao G, Li CY and Wei L: KOBAS 2.0: A web server for annotation and identification of enriched pathways and diseases. Nucleic Acids Res 39: W316-W322, 2011.

16. Wang J, Duncan D, Shi Z and Zhang B: WEB-based GEne SeT AnaLysis Toolkit (WebGestalt): Update 2013. Nucleic Acids Res 41: W77-W83, 2013.

17. Lamb J: The Connectivity Map: A new tool for biomedical research. Nat Rev Cancer 7: 54-60, 2007.

18. Pufe T, Petersen WJ, Miosge N, Goldring MB, Mentlein R, Varoga DJ and Tillmann BN: Endostatin/collagen XVIII-an inhibitor of angiogenesis-is expressed in cartilage and fibrocartilage. Matrix Biol 23: 267-276, 2004.

19. Mogili NS, Krishnaswamy VR, Jayaraman M, Rajaram R, Venkatraman A and Korrapati PS: Altered angiogenic balance in keloids: A key to therapeutic intervention. Transl Res 159: 182-189, 2012.

20. Deshpande M, Papp S, Schaffer L and Pouyani T: Hydrocortisone and triiodothyronine regulate hyaluronate synthesis in a tissue-engineered human dermal equivalent through independent pathways. J Biosci Bioeng 119: 226-236, 2015.

21. Bolós V, Grego-Bessa J and de la Pompa JL: Notch signaling in development and cancer. Endocr Rev 28: 339-363, 2007.

22. Syed F and Bayat A: Notch signaling pathway in keloid disease: Enhanced fibroblast activity in a Jagged-1 peptide-dependent manner in lesional vs. Extralesional fibroblasts. Wound Repair Regen 20: 688-706, 2012.

23. Ehrlich HP, Desmoulière A, Diegelmann RF, Cohen IK, Compton CC, Garner WL, Kapanci Y and Gabbiani G: Morphological and immunochemical differences between keloid and hypertrophic scar. Am J Pathol 145: 105-113, 1994.

24. Kischer CW, Thies AC and Chvapil M: Perivascular myofibroblasts and microvascular occlusion in hypertrophic scars and keloids. Hum Pathol 13: 819-824, 1982.

25. Nör JE, Christensen J, Mooney DJ and Polverini PJ: Vascular endothelial growth factor (VEGF)-mediated angiogenesis is associated with enhanced endothelial cell survival and induction of Bcl-2 expression. Am J Pathol 154: 375-384, 1999.

26. Gira AK, Brown LF, Washington CV, Cohen C and Arbiser JL: Keloids demonstrate high-level epidermal expression of vascular endothelial growth factor. J Am Acad Dermatol 50: $850-853,2004$

27. Fujiwara M, Muragaki Y and Ooshima A: Upregulation of transforming growth factor-betal and vascular endothelial growth factor in cultured keloid fibroblasts: Relevance to angiogenic activity. Arch Dermatol Res 297: 161-169, 2005.

28. Javad F, Marriage F, Bayat A and Day PJ: Perturbation of cell cycle expression in keloid fibroblast. Skinmed 10: 152-159, 2012.

29. Huang L, Wong Y, Cai Y, Lung I, Leung C and Burd A: Low-dose 5-fluorouracil induces cell cycle G2 arrest and apoptosis in keloid fibroblasts. Br J Dermatol 163: 1181-1185, 2010 .

30. Lane DP: The regulation of p53 function: Steiner Award Lecture. Int J Cancer 57: 623-627, 1994. 
31. Tanaka A, Hatoko $M$, Tada H, Iioka $H$, Niitsuma $K$ and Miyagawa S: Expression of p53 family in scars. J Dermatol Sci 34: 17-24, 2004.

32. Lukert BP and Raisz LG: Glucocorticoid-induced osteoporosis. Rheum Dis Clin North Am 20: 629-650, 1994.

33. Wichers M, Springer W, Bidlingmaier F and Klingmüller D: The influence of hydrocortisone substitution on the quality of life and parameters of bone metabolism in patients with secondary hypocortisolism. Clin Endocrinol (Oxf) 50: 759-765, 1999.

34. Langdahl BL, L økke E, Carstens M, Stenkjær LL and Eriksen EF: Osteoporotic Fractures Are Associated with an 86-Base Pair Repeat Polymorphism in the Interleukin-1-Receptor Antagonist Gene But Not with Polymorphisms in the Interleukin-1beta Gene. J Bone Miner Res 15: 402-414, 2000.

35. Kimble RB, Vannice JL, Bloedow DC, Thompson RC, Hopfer W, Kung VT, Brownfield $C$ and Pacifici R: Interleukin-1 receptor antagonist decreases bone loss and bone resorption in ovariectomized rats. J Clin Invest 93: 1959-1967, 1994.
36. Chen HY, Chen WC, Wu MC, Tsai FJ and Lin CC: Interleukin-1beta and interleukin-1 receptor antagonist gene polymorphism in postmenopausal women: Correlation to bone mineral density and susceptibility to osteoporosis. Maturitas 44: 49-54, 2003.

37. Mann V and Ralston SH: Meta-analysis of COL1A1 Sp1 polymorphism in relation to bone mineral density and osteoporotic fracture. Bone 32: 711-717, 2003.

38. Viguet-Carrin S, Garnero P and Delmas P: The role of collagen in bone strength. Osteoporos Int 17: 319-336, 2006.

39. Sun Y, Sun Q, Fan C, Shen J, Zhao W, Guo Y, Su T, Wang W, Ning $G$ and Bian L: Diagnosis and therapy for Cushing's disease with negative dynamic MRI finding: A single-centre experience. Clin Endocrinol (Oxf) 76: 868-876, 2012

40. Bori Segura G, Torres y Gutierrez Rubio A, Herrera Gómez LE and Olguín Uribe J: Efficacy and tolerability of acemetacin, a non-steroidal anti-inflammatory drug, in Mexican patients: Result of the ETAPAM Study. Proc West Pharmacol Soc 45: 104-107, 2002. 